\title{
Correction to: Penalized Supervised Star Plots: Example Application in Influenza-Specific CD4+ T Cells, by Holmes TH, Subrahmanyam PB, Wang W, and Maecker HT. Viral Immunol 2019;32(2):102-109. DOI: 10.1089/vim.2018.0046
}

N The March 2019 issue of Viral Immunology (vol. 32, no. 2; pages 102-109), the article titled, Penalized Supervised Star
Plots: Example Application in Influenza-Specific CD4+ T Cells by Tyson H. Holmes et al., requires a correction to the Supplementary Figure S5 legend.

The fourth sentence in the Supplementary Figure S5 legend reads:

"Insert shows importance value r for each discriminant function."

The sentence should have appeared as:

“Insert shows Discriminant Analysis eigenvalues."

The online version of the article has been corrected to reflect this change.

This error was made by the first author (T.H.H.), who apologizes for the mistake and any inconvenience caused. 